\title{
Le vieillissement des Internes de Cuve - Programme de recherche en support à la durée de fonctionnement des réacteurs REP
}

\author{
Benoit Tanguy ${ }^{1}$, Faiza Sefta ${ }^{2}$, P. Joly ${ }^{3}$ \\ ${ }^{1}$ Service d'Etude des Matériaux Irradiés \\ DEN/DANS/DMN/SEMI \\ CEA Saclay, 91191 Gif sur Yvette cedex \\ Tel : 0169089420, e-mail : benoit.tanguy@cea.fr \\ ${ }^{2}$ EDF R\&D Les Renardières \\ Route de Sens - Ecuelles \\ 77250 Moret-sur-Loing, France \\ Tel : 0169089420, e-mail : faiza.sefta@edf.fr \\ ${ }^{3}$ Engineering and Project (DIP) - AREVA \\ Tour AREVA \\ 92084 Paris La Défense cedex - France \\ Tel : 0134967217, e-mail : pierre.joly@areva.com
}

\section{1- Introduction}

Les aciers inoxydables austénitiques, en raison de leur propriété de résistance à la corrosion sont très utilisés pour les éléments de structures des réacteurs nucléaires soumis à un milieu chimiquement agressif. Différentes nuances de ces aciers sont notamment largement employées dans les réacteurs à eau légère (REL) constituant la majorité du parc électronucléaire mondial mais sont aussi envisagées pour des systèmes futurs tels que ITER où ils seront potentiellement soumis à des conditions de fonctionnement plus sévères (température, flux, dose, pression). Dans les réacteurs à eau pressurisée (REP), les structures d'internes de cœur (Figure 1) ont notamment pour rôle de porter le poids du cœur, de maintenir en alignement les assemblages combustibles, les grappes de commande et l'instrumentation, de canaliser l'écoulement du fluide caloporteur et de protéger la cuve contre les rayonnements émis par l'irradiation. Au cours du fonctionnement du réacteur, les structures d'internes (Figure 1) en aciers inoxydables austénitiques 304L hypertrempé $(18 \% \mathrm{Cr}, 10 \% \mathrm{Ni})$ pour les cloisons et renforts et en 316 écroui $(17 \% \mathrm{Cr}, 11 \% \mathrm{Ni}, 2.5 \% \mathrm{Mo})$ pour les vis, sont soumises à la combinaison des rayonnements neutroniques et de températures élevées (300 à $370^{\circ} \mathrm{C}$ ). Pour une durée de fonctionnement de 40 ans, ces structures auront subies des doses de plusieurs dizaines de dpa (déplacement par atome) (voir Tableau 1).

\begin{tabular}{|c|c|c|c|c|}
\hline Component & Material & $\begin{array}{l}\text { Temperature } \\
\text { (C) }\end{array}$ & $\begin{array}{l}\text { Dose à } 40 \\
\text { ans }^{*}(\mathrm{dpa})\end{array}$ & $\begin{array}{c}\text { Dose à } 60 \\
\text { ans }^{* \star} \text { (dpa) }\end{array}$ \\
\hline Vis de liaison cloison-renfort & $\begin{array}{c}\text { CW-316 and } 316 \mathrm{~L} \\
17 \% \mathrm{Cr}-11 \% \mathrm{Ni}-2.5 \% \mathrm{M}\end{array}$ & $\sim 300$ to 350 & up to -80 & $\max 120$ \\
\hline Plaques de cloisonnement & \multirow{3}{*}{$\begin{array}{c}\text { SA-304L } \\
18 \% \mathrm{Cr}-10 \% \mathrm{Ni}\end{array}$} & -300 to 350 & up to -80 & $\max 120$ \\
\hline Renforts & & $\sim 300$ to 350 & up to $\sim 50$ & $\max 75$ \\
\hline Enveloppe de coeur & & $\sim 300$ & up to $\sim 10$ & $\max 15$ \\
\hline Soudures enveloppe de coeur & 308L welds (SAW) & -300 & up to $\sim 10$ & $\max 15$ \\
\hline
\end{tabular}


Tableau 1: Caractéristiques d'irradiation (Temp, dose) pour les différents composants d'Internes pour des durées de fonctionnement de 40 et 60 ans.

L'irradiation neutronique des aciers inoxydables austénitiques dans le domaine de fonctionnement des REP (température, dose, flux, ...) provoque une évolution microstructurale et une évolution des propriétés mécaniques. Par ailleurs, l'exposition à un environnement corrosif (fluide primaire) combinée aux modifications microstructurales du matériau par l'irradiation entraine une sensibilité à la fissuration par corrosion sous contrainte dite «assistée par l'irradiation» (IASCC) qui affecte essentiellement les vis de cloison du palier CP0. Le gonflement sous irradiation qui pourrait résulter de la formation de nanocavités est un dommage potentiel qu'il est important de maitriser dans la perspective d'une durée de fonctionnement au-delà de 40 ans.

Les structures d'internes sont des composants «remplaçables », cependant compte tenu de leur rôle dans la durabilité et de la difficulté liée au remplacement de l'ensemble des internes, l'évaluation du comportement sous irradiation des Internes de cuve représente un enjeu économique majeur pour le Parc. Le vieillissement des Internes de cuve doit être géré pour optimiser l'exploitation du réacteur et minimiser le nombre et la durée des arrêts de tranche. L'acquisition des connaissances sur les mécanismes de vieillissement des matériaux des internes inférieurs sous irradiation en conditions REP (ensemble cloison-renfort pour les paliers 900 et $1300 \mathrm{MWe}$ et réflecteur lourd pour l'EPR), sur les effets de ce vieillissement sur les propriétés des matériaux et sur ses conséquences en termes de dégradations est réalisée notamment dans le cadre du projet de recherche, «Internes de cuve », collaboratif dédié entre EDF, AREVA et le CEA. D'autres programmes nationaux et internationaux, tels que les programmes européens (PERFECT, PERFORM-60 [1]) avec des travaux plus amont, complètent la compréhension des mécanismes de vieillissement de ces matériaux. Le projet «Internes de cuve » vise à apporter des éléments pour alimenter les dossiers de sûreté analysant la durée de vie des internes de cuve REP et les dossiers nécessaires à la démonstration de l'aptitude des composants principaux à assurer leur fonction jusqu'à la VD4 + 20 ans pour les paliers 900, 1300 MWe et N4. Pour cela, le projet contribue à l'amélioration de la connaissance des mécanismes de dégradation sous irradiation et à l'acquisition de données matériaux :

$>$ en menant des actions de compréhension des mécanismes de dégradation des matériaux dus à l'irradiation (évolution microstructurale des matériaux irradiés, IASCC, gonflement, durcissement, fluage d'irradiation, ...) ;

$>$ en améliorant les lois de comportement utilisées par l'ingénierie des aciers inoxydables austénitiques irradiés et sous irradiation ;

$>$ en améliorant le critère de fissuration par IASCC fonction des paramètres de fonctionnement (température, dommage d'irradiation, contrainte, ...) ;

$>$ en étant présent dans les programmes expérimentaux internationaux et en menant une veille scientifique. 

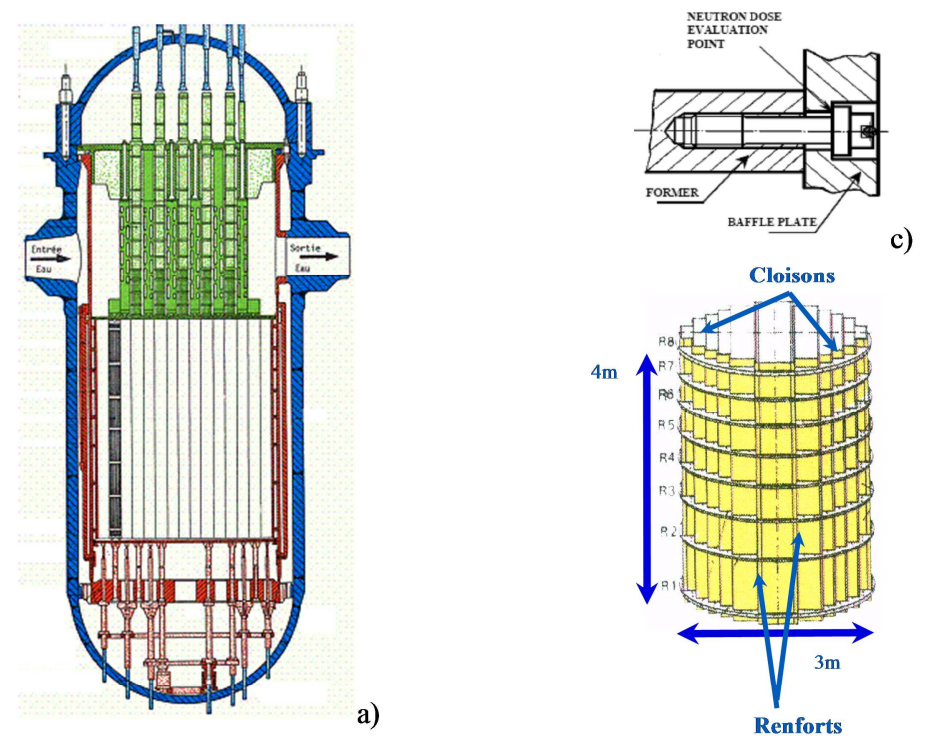

b)

Figure 1 : Structures des internes de cour en acier inoxydable austénitique dans un réacteur REP. a) Vue générale du réacteur, b) Renforts et cloisons en 304L hypertrempé, c) Vis de liaison renfort/cloison en 316 écroui.

Cet article a pour objectif de donner un aperçu non-exhaustif du programme de recherche collaboratif sur le vieillissement des matériaux des internes de Cuve mené dans le cadre de l'Institut tripartite CEA, EDF AREVA. Dans une première partie (section 2), les différents phénomènes de vieillissement, identifiés ou potentiels seront décrits. La méthodologie générale mise en œuvre pour identifier puis conduire les actions de R\&D sera ensuite donnée dans la deuxième partie (section 3). La troisième partie sera dédiée à une description plus détaillée des programmes liés à certains phénomènes. On s'intéressera à l'IASCC, au fluage sous irradiation et au gonflement. Enfin, les principales conclusions et les perspectives seront données dans la dernière partie.

\section{2- Phénomènes de Vieillissement sous irradiation des matériaux d'Internes de Cuve}

Les matériaux d'Internes de Cuve sont des aciers austénitiques inoxydables de structure cubique à faces centrées. D'une manière générale, l'irradiation provoque un durcissement par une augmentation de la limite d'élasticité, et une diminution de la ductilité des aciers austénitiques (Figure 2). Pour les faibles doses, jusqu'à une dizaine de dpa, et dans le domaine de température d'intérêt $\left(290^{\circ} \mathrm{C}-350^{\circ} \mathrm{C}\right)$, le matériau montre une forte augmentation de la limite d'élasticité s'accompagnant d'une perte marquée de ductilité. Au-delà de $10 \mathrm{dpa}$, une saturation de ces effets est observée. La forte diminution de la ductilité due à l'irradiation a une implication significative sur la diminution de la ténacité dans les aciers austénitiques. Le durcissement résulte de la création d'amas de défauts sous irradiation (boucle de dislocations, précipités, cavités, ...) qui font obstacles au mouvement des dislocations. La contrainte à exercer pour assurer la mobilité des dislocations augmente. Pour les aciers inoxydables austénitiques, aux températures d'irradiation rencontrées dans les REP, les principaux défauts sont les boucles de dislocations interstitielles, les cavités (et/ou bulles de gaz) et la précipitation induite par l'irradiation (notamment de type $\mathrm{Ni}_{3} \mathrm{Si}$ ). Les mécanismes de plasticité 
sont également affectés avec la formation de canaux de localisation de la déformation appelés bandes claires.

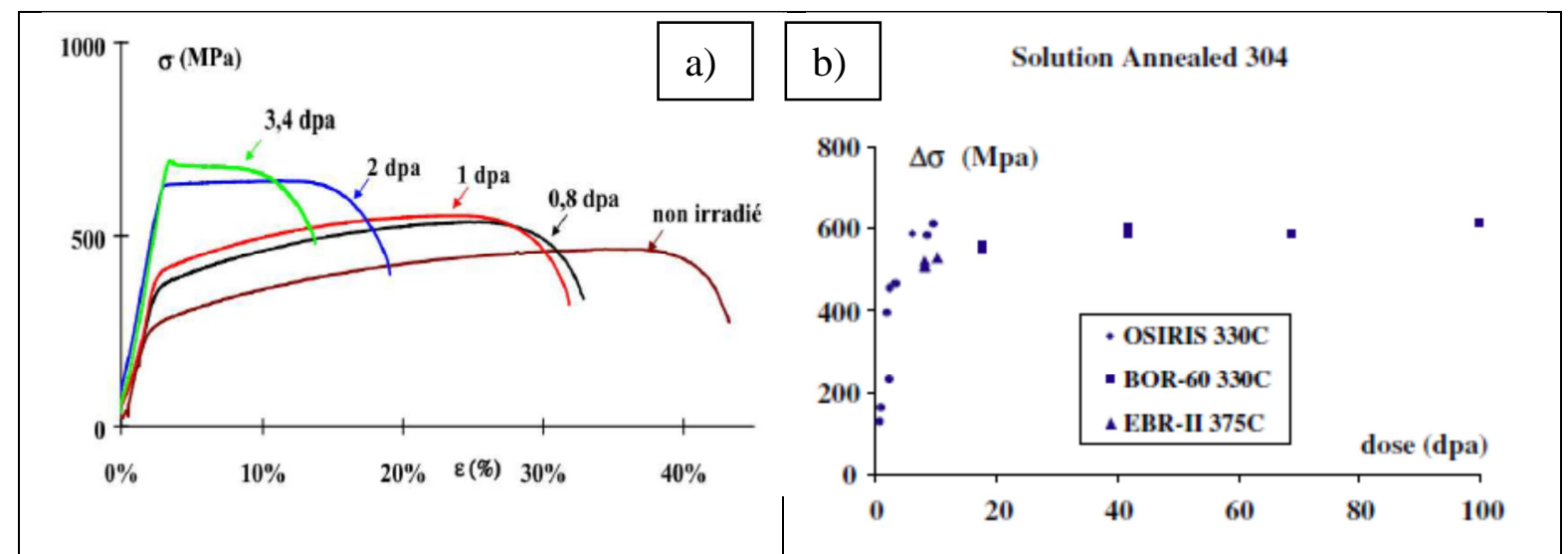

Figure 2 : Evolution des propriétés de traction avec la dose pour une nuance 304 état hypertrempé irradiée et testée à $330^{\circ} \mathrm{C}$ [9] et saturation du durcissement au-delà de 10-20 dpa [10].

Sous flux neutronique, bien que ces matériaux ne soient pas ou peu sensibles au fluage thermique (déformation dépendante du temps pour une contrainte inférieure à la limite d'élasticité) aux températures d'intérêt, le phénomène de fluage d'irradiation apparait. Ces caractéristiques sont très différentes de celles du fluage thermique avec un exposant de la contrainte proche de l'unité et une faible dépendance en température. Il existe différents régimes de fluage sous irradiation : après un régime transitoire de courte durée (autour de 1 dpa), le matériau entre dans un régime stationnaire ou la déformation de fluage est proportionnelle à la dose et à la contrainte. Il est connu que l'apparition du gonflement observée à plus hautes températures dans les irradiations dans les réacteurs à neutrons rapides (RNR) conduirait au troisième régime caractérisé par une accélération de la cinétique de fluage. Bien que les données expérimentales soient disponibles et intégrées dans le paramétrage des lois matériaux pour l'ingénierie, les mécanismes du fluage d'irradiation induits par les défauts ponctuels ne sont pas entièrement établis. Le plus classique des mécanismes avancés est le fluage APIC (montée des dislocations par absorption préférentielle induite selon la contrainte). Il a été montré récemment $[2,3]$ par la modélisation de ce mécanisme par la technique de la dynamique d'amas que les cinétiques obtenues de la déformation en fonction de la dose étaient très inférieures à celles mesurées expérimentalement mettant ainsi en évidence qu'un autre mécanisme entre en jeux. A partir de la modélisation, le rôle des joints de grains apparait comme un mécanisme potentiel qui permet de reproduire le bon ordre de grandeur des cinétiques mesurées et leur sensibilité à la contrainte. Le fluage par irradiation a pour principale conséquence une relaxation des contraintes au niveau des vis de liaison [4] et est donc susceptible d'améliorer le comportement à la corrosion sous contrainte assistée par l'irradiation (IASCC) [5]. Ce phénomène, qui s'est traduit dans les REP (majoritairement le palier $900 \mathrm{MW} \mathrm{CP0),} \mathrm{par} \mathrm{une}$ fissuration de quelques vis de liaison cloison -renfort (Figure 3), résulte d'un ensemble complexe de phénomènes affectant le matériau et les phénomènes d'oxydation résultant de l'exposition au fluide primaire encore incomplètement compris.

Bien que n'étant pas le seul facteur intervenant dans la sensibilité à l'IASCC, la ségrégation intergranulaire induite par l'irradiation (RIS - Radiation Induced Segregation) en modifiant la chimie des joints de grains, notamment par un appauvrissement en chrome et par un enrichissement en silicium a été identifié comme un acteur de la fragilisation intergranulaire. 
Les profils de ségrégation évoluent avec l'augmentation de la dose. Cette ségrégation intergranulaire évolue rapidement jusqu'à des doses autour de 5 dpa puis sature ou augmente très lentement. L'apparition de ces différentes modifications de la microstructure (boucle de dislocation, RIS, ...)se produisant à des niveaux de fluence similaires, il est difficile de séparer leur contribution individuelle dans le mécanisme d'IASCC, de plus, d'autres facteurs influents pourraient apparaitre pour des niveaux élevés de fluence. Enfin, il est possible que les effets de synergie entre les différents facteurs impliqués puissent évoluer en fonction du domaine de fluence considéré.

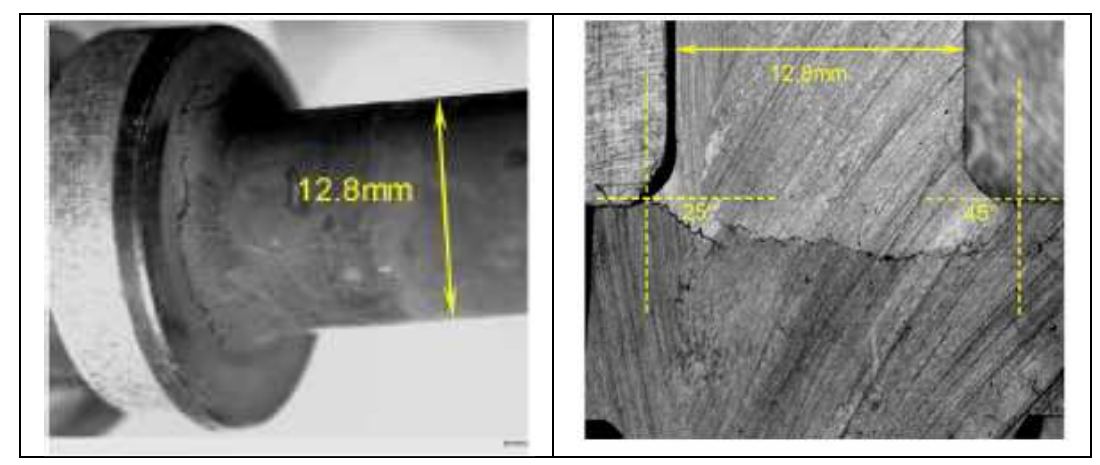

\section{Figure 3 : Illustration de la fissuration de vis de liaison renfort-paroi par IASCC [6]}

Le retour d'expérience des études liées aux structures internes des réacteurs à neutrons rapides montre que la sursaturation des lacunes crées par l'irradiation peut conduire à la germination et à la croissance de cavités dont la signature macroscopique est une expansion volumique isotrope appelée gonflement. Ce phénomène est un phénomène à seuil : après une période d'incubation (phase de germination des cavités), le gonflement évolue linéairement avec la dose (phase de croissance des cavités). La dépendance du gonflement vis-à-vis de la dose, de la température, du flux de neutrons et de la quantité de gaz crée par l'irradiation fait que le REX des études sur les réacteurs rapides ne peut être transposé aux REPs. L'anticipation du phénomène de gonflement nécessite donc de conduire des irradiations en conditions représentatives de celle des REP et de développer des outils de simulations d'évolution de la microstructure à bases physiques permettant d'appréhender l'effet des différents paramètres d'irradiation et métallurgiques.

\section{3- Méthodologie des études de R\&D}

Afin d'appréhender les phénomènes de vieillissement par irradiation liés aux Internes, les études R\&D s'organisent autour de 3 axes principaux que sont (i) la détermination des conditions d'apparitions des phénomènes de dégradation, (ii) l'évaluation des effets des phénomènes de vieillissement sur la tenue des composants internes, et (iii) la compréhension des mécanismes des phénomènes de vieillissement. Le premier axe s'appuie sur l'analyse de REX des composants déposés et sur des irradiations en réacteurs expérimentaux. En France, le réacteur OSIRIS du CEA, dont le spectre d'irradiation est représentatif des REPs est utilisé pour ces études. Pour atteindre des doses plus importantes, représentatives de 60 ans de fonctionnement REP pour certains composants (voir Tableau 1), on utilise également des réacteurs à spectre rapide tel que le réacteur BOR-60 en Russie qui permet d'anticiper grâce à un flux neutroniques 10 fois plus importants les phénomènes de vieillissement jusqu'à 120 dpa en quelques années. L'intérêt de ces irradiations est la bonne maitrise de leurs conditions neutroniques. Pour évaluer la représentativité des irradiations faites dans les RNR, on les confronte aux basses doses aux données obtenues à partir des irradiations provenant de REP 
ou d'OSIRIS. Pour les structures d'Internes, l'évaluation des effets du vieillissement sur la tenue des composants est évaluée à parti de calcul de structure qui s'appuient sur la loi de comportement du matériau fonction de l'irradiation [7] qui prend en compte l'effet du fluage d'irradiation et du gonflement sur la déformation mécanique du composant. Dans le cas des vis de liaisons paroi-renfort, les contraintes résultantes au niveau des vis sont comparées à un critère empirique d'amorçage en IASCC établi à partir de nombreux essais à l'international et notamment en France dans les laboratoires chauds (LECI) du CEA Saclay [8]. Enfin, parallèlement à ces études qui alimentent pour chaque période décennale les dossiers de justification de fonctionnement de ces structures, des études de compréhension et de modélisation amonts sont conduites afin, à terme, d'anticiper le vieillissement des matériaux de ces structures. Ces études théoriques et expérimentales s'appuient sur des irradiations analytiques permettant d'étudier de manière isolée certains phénomènes et sur des observations fines de la microstructure d'irradiation avec des outils tels que la microscopie électronique en transmission et la sonde atomique tomographique (SAT) qui permettent des échelles d'observation de l'ordre du nanomètre. Enfin, il faut noter au niveau international et notamment européen avec les projets PERFECT et PERFORM-60 [1] du PCRD-7 un effort continu depuis une dizaine d'année pour développer des outils de simulation prédictifs des effets de l'irradiation sur les matériaux d'Internes et de cuve à différentes échelles.

\section{4- Programmes d'études des phénomènes de dégradation}

Cette partie est dédiée à une description plus détaillée mais non-exhaustive des programmes de recherche en support à la durée de fonctionnement des réacteurs REP. On s'intéresse à l'IASCC, au fluage sous irradiation et au gonflement.

\section{a. Fluage sous irradiation}

Sous flux neutronique, soumis à une charge inférieure à la limite d'élasticité, les aciers d'Internes montrent une déformation qui évolue avec le temps alors qu'ils ne sont pas ou peu sensibles au fluage thermique pour ces températures $\left(290^{\circ} \mathrm{C}-350^{\circ} \mathrm{C}\right)$. Pratiquement, le REX montre une diminution du couple de serrage des vis avec la dose [4] qui traduit l'apparition sous flux neutronique du phénomène de fluage d'irradiation. La connaissance des lois gouvernant les cinétiques de fluage sous irradiation (on parle dans le cas des vis de relaxation sous irradiation, la déformation $\mathrm{du}$ vis étant imposée lors du serrage initial) est particulièrement importante d'une part pour pouvoir évaluer, en fonction de la dose, la fonctionnalité des vis et, d'autre part pour pouvoir évaluer en fonction de la dose le niveau de contrainte auquel est soumis le composant. Ce niveau de contrainte sera utilisé, notamment pour évaluer la situation des vis vis-à-vis d'un amorçage par IASCC. Un premier programme de recherche a été conduit pour quantifier l'évolution du fluage d'irradiation en fonction de la dose pour des contraintes entre 127 et $220 \mathrm{MPa}$. Pour cela, des tubes pressurisés (Figure 4a) ont été irradiés dans trois réacteurs, deux de type RNR et OSIRIS (spectre mixte à des doses entre 10 et $120 \mathrm{dpa}$ ). La mesure de l'évolution diamétrale des tubes avec la dose (Figure 4c) a permis de valider le type de loi de fluage $\left(\bar{\varepsilon}=A_{1}(\bar{\sigma} \phi-\eta)\right.$ pour $\phi \sigma>\eta$ ou $\sigma$ est la contrainte, $\phi$ la dose, $\eta$ la dose d'incubation et $A$ une constante qui dépend du matériau et du flux) et d'en déterminer les paramètres. Il a ainsi été montré (i) une différence significative des cinétiques de fluage d'irradiation entre le 316 écroui (vis) et le 304 hypertrempé (paroi, renfort), le dernier fluant plus rapidement, (ii) la présence d'une dose d'incubation. Ce modèle a été utilisé avec un accord satisfaisant pour évaluer l'évolution des couples de serrage avec la dose lors d'une campagne de remplacement de vis sur 3 réacteurs REP français (figure 4d) confirmant que l'évolution des couples de serrage des vis est lié au fluage d'irradiation. 
Actuellement, un second programme est en cours dans le réacteur OSIRIS pour des niveaux de contrainte plus élevés qui sont obtenus à partir de lame en flexion (Figure 4b).

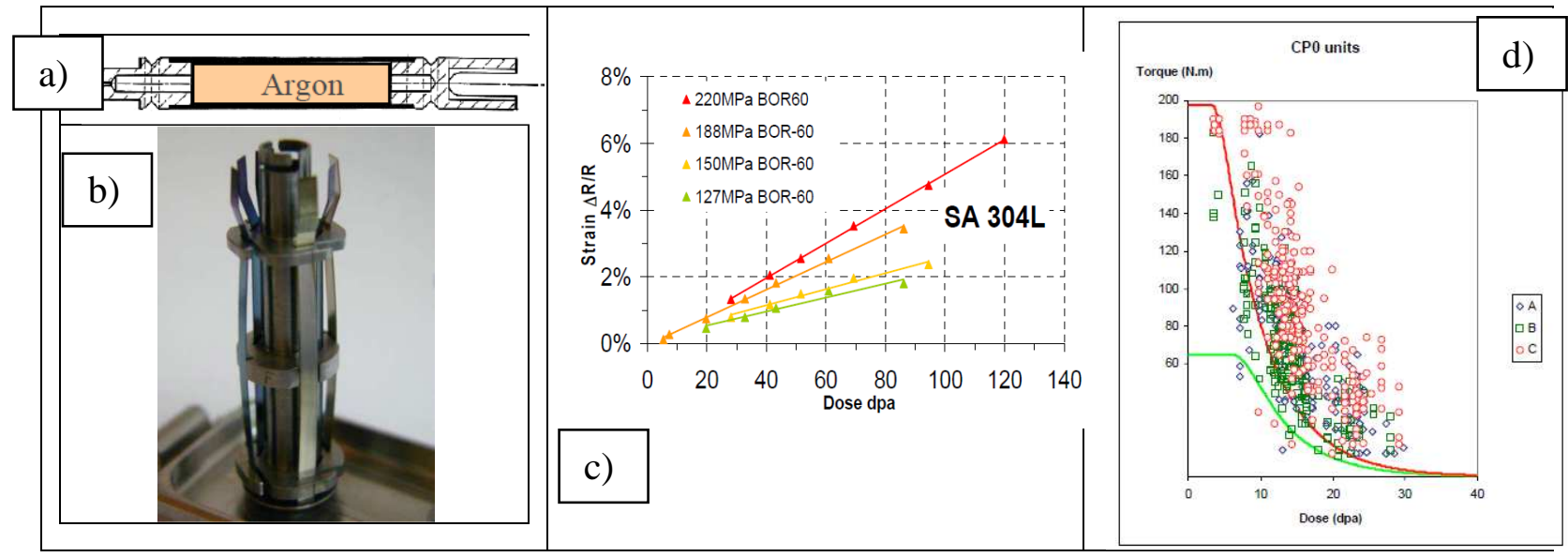

Figure 4 : Etude expérimentale du fluage d'irradiation. a)tube pressurisée permettant d'obtenir une contrainte appliquée constante, b) bobines avec des lames en flexion, c) Résultats de mesure sur tubes pressurisé à différents niveaux de contraintes, d) mesures expérimentales de couple de desserrage des vis de liaison paroi-renfort en fonction la dose et comparaison avec la modélisation (traits continus pour un couple initial minimum et maximum).

\section{b. Gonflement}

Le REX actuel des expertises de composants REP déposés [6, 11] montre que les évolutions microstructurales constatées sur ces composants ne conduisent pas à un gonflement macroscopique significatif. Toutefois, le gonflement induit par l'irradiation est considéré comme un phénomène potentiel pouvant limiter la durabilité de ces structures pour les fortes doses, essentiellement du fait des modifications dimensionnelles qui en seraient induites (gradient de gonflement entre différents composants). Compte tenu des forts niveaux de fluence auquel le gonflement macroscopique est associé en réacteurs à neutrons rapides (RNR), ce phénomène a été peu étudié expérimentalement en environnement REP. La plupart des études ont été menées dans les RNR où les conditions de température, de spectre neutronique et de doses reçues sont plus propices au développement du gonflement. L'évaluation du risque de gonflement en conditions REP pour des durées de fonctionnement de 60 ans a été entreprise par le programme expérimental international GONDOLE en cours dans le réacteur OSIRIS du CEA dont les caractéristiques d'irradiation (spectre, température d'irradiation) sont proches de celles des REP. Afin de quantifier la présence significative de gonflement pour des forts niveaux de fluence d'intérêt et également d'évaluer les cinétiques (fluence seuil, vitesse de gonflement), des plaquettes de matériaux vierges et pré-irrradiés jusqu'à 80 dpa en conditions REP ont été placés dans le réacteur. A l'issue de l'irradiation, qui aura duré plus de 9 ans permettant d'accumuler plus de 30 dpa dans le réacteur dans des conditions d'irradiations bien connues, l'évaluation du gonflement macroscopique sera basée sur des matériaux avec des doses comprises entre 30 et 110 dpa. Plusieurs nuances d'aciers inoxydables austénitiques avec différents états métallurgiques (hypertrempé et écroui) et avec différentes teneur en éléments d'addition chimiques ont été mises en irradiation afin de pouvoir appréhender l'effet de ces éléments sur l'apparition d'un gonflement macroscopique.

Parallèlement à ce programme, des études de compréhension des mécanismes pouvant conduire à la formation et la croissance des amas lacunaires sont conduites. L'évolution de 
microstructure d'irradiation et spécifiquement des amas lacunaires et d'interstitiels est étudié avec la modélisation de la dynamique d'amas qui permet de simuler la germination, la croissance et la coalescence des amas de solutés ou de défauts sur des temps comparables aux durées expérimentales. Le code CRESCENDO, co-développé par le CEA et EDF est ainsi utilisé pour pouvoir appréhender l'effet de différents paramètres (flux, taux de gaz produit, température) sur le gonflement résultant des amas lacunaires. (Figure 5)

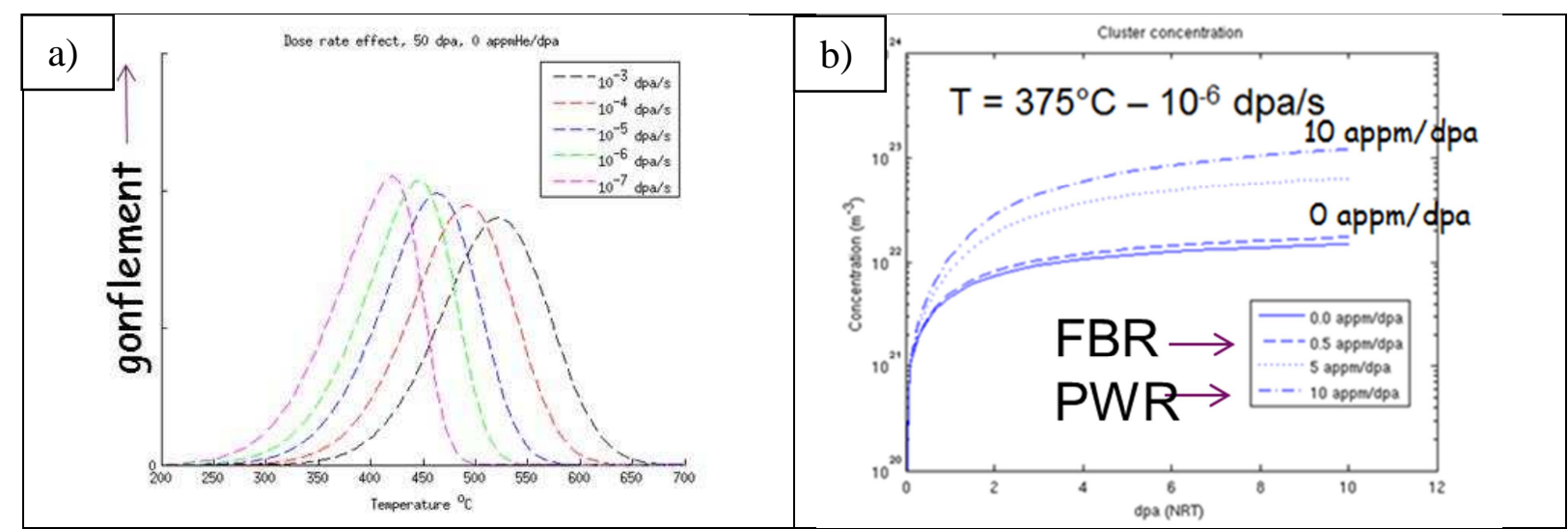

Figure 5: Etude paramétrique sur le gonflement calculé à partir des amas lacunaires par le code CRESCENDO. a)effet du flux sur la température du pic de gonflement, b) effet du taux d'Hélium sur la concentration des amas lacunaires. (Courtesy A. Courcelle, CEA)

\section{c. IASCC (Fissuration sous contrainte assistée par l'irradiation)}

La synergie des mécanismes impliqués dans l'endommagement par IASCC a conduit à définir une stratégie d'étude à plusieurs niveaux, tant au niveau expérimental qu'au niveau de la modélisation.

Au niveau expérimental, les études réalisées peuvent être classifiées en trois catégories: (i) essais de CSC in-situ en réacteurs d'essais, (ii) essais de CSC post-irradiation sur matériaux irradiés aux neutrons, (iii) essais de CSC sur matériaux "représentatifs" ou «modèles », ces derniers sont des matériaux qui sont susceptibles de représenter une des modifications microstructurales dus à l'irradiation tel que le durcissement, la ségrégation chimique intergranulaire ou la localisation de la déformation plastique. La complexité et le coût des essais et de leur interprétation va en diminuant de la première catégorie à la dernière. La première catégorie est la plus représentative des effets de synergie se produisant dans les réacteurs industriels, la représentativité de l'historique de chargement restant à ce jour le paramètre le moins reproductible Les études paramétriques sont généralement menées sur des matériaux "représentatifs". De nombreuses études ont été menées sur des matériaux de laboratoire avec une ségrégation artificielle ou sur des nuances industrielles avec un durcissement obtenu par écrouissage à froid équivalents à ceux observés sur matériau irradié. Toutes ces études sont menées dans des boucles de corrosion qui permettent de simuler l'environnement du milieu pour les REP ou/et pour les REB. La représentativité ${ }^{1}$ de la microstructure des aciers austénitiques inoxydables irradiés avec des particules énergétiques (protons, ions) vis-à-vis de celle obtenue par irradiation neutronique a permis, compte tenu de la faible activité des échantillons irradiés avec ces particules, le développement de nombreuses études paramétriques ces dernières années.

\footnotetext{
${ }^{1}$ Cette représentativité est basée sur la population des boucles de Franck, la ségrégation induite par l'irradiation et la sensibilité à la CSC. Il faut cependant noter que les phénomènes physiques résultant des interactions entre le réseau atomique et les deux types de particules sont différents.
} 
Elles ont notamment permis de mettre en évidence le rôle de la composition chimique initiale et du niveau des impuretés sur la ségrégation et le rôle de la localisation de la déformation plastique sur l'IASCC se produisant lors de sollicitations dynamiques post-irradiation en milieu REB [12]. Ces études, qui ont permis d'isoler des facteurs prépondérants, restent cependant limitées à des niveaux moyens de dose (2-10 dpa) et permettent uniquement l'étude de l'amorçage compte-tenu des profondeurs d'irradiation de l'ordre de la trentaine de micromètres.

La difficulté et le coût des essais sur matériaux irradiés aux neutrons conduisent à réaliser ce type d'essais pour établir un critère empirique d' amorçage en IASCC (en REP), quantifier des cinétiques de propagation (REB), et comprendre l'effet de paramètres spécifiques (température, chimie, transitoire). Ces essais sont majoritairement conduits post-irradiation dans une boucle de corrosion en laboratoire chaud, telle que celle disponible au LECI du CEA Saclay, sur des matériaux pré-irradiés en réacteurs industriels ou en réacteurs expérimentaux. L'établissement d'une courbe limite de sensibilité à l'amorçage par IASCC (Figure 6b) en conditions quasi-statiques, plus représentatives de celles des vis de cloisons, et de son évolution en fonction de différents paramètres (température, milieu, nuance, etc) est un des principaux objectifs de ce type d'essais pour évaluer empiriquement le risque de fissuration. Ces essais ne permettent cependant pas d'appréhender le rôle de la relaxation des contraintes et du fluage d'irradiation dans le mécanisme d'IASCC. Ces effets ne peuvent être appréhendés que par des essais de CSC sous flux en réacteurs expérimentaux qui nécessitent une très grande maitrise technologique. Du fait de leur complexité et des équipements spécifiques inhérents aux essais de CSC sous flux, seuls quelques réacteurs expérimentaux tels que celui de l'OCDE à Halden (Norvège), le réacteur japonais JMTR à Oarai et celui du NRI à Rez (République Tchèque) ont la capacité de mener ce type d'essai in-situ. La réalisation d'essais d'amorçage ou de propagation nécessite des montages de mise en charge et une instrumentation spécifiques. A ce jour, la réalisation d'essai de CSC in-situ n'est plus possible en France et est envisagée parmi les équipements du futur réacteur d'essais international RJH qui est en construction au CEA.

La comparaison du critère de fissuration en IASCC, aux contraintes évaluées au niveau des vis et la localisation de l'amorçage est montré sur la Figure 6c.

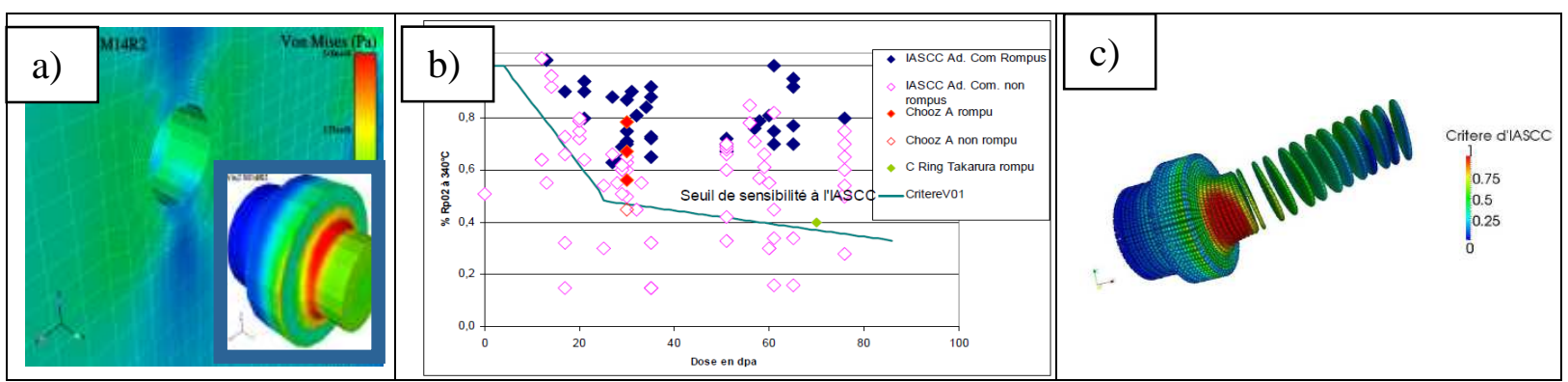

Figure 6 : Evaluation de l'amorçage en IASCC. a) Evaluations des contraintes au niveau d'un vis, b)critère d'amorçage en IASCC, c) Evaluation et localisation du risque de fissuration.

Au niveau de la modélisation, les efforts actuels portent essentiellement sur l'amorçage. Une première tentative est l'établissement d'un critère semi-empirique de sensibilité à l'IASCC, qui devra notamment considérer la modification des mécanismes de déformation, la teneur en éléments chimiques, les cinétiques d'oxydation et leur évolution avec la dose. Parallèlement, une stratégie de modélisation multi-échelles a été mise en place dans les projets européens PERFECT puis PERFORM-60. L'objectif est une meilleure compréhension du mécanisme de l'IASCC à partir de la modélisation de la microstructure, de l'oxydation et de la réponse 
mécanique du matériau et de leur évolution sous flux afin de pouvoir proposer un critère de fissuration.

\section{5- Conclusions et perspectives}

Les structures d'Internes de Cuve ont pour rôle de porter le poids du cœur, de maintenir en alignement les assemblages combustibles, les grappes de commande et l'instrumentation, de canaliser l'écoulement du fluide caloporteur et de protéger la cuve contre les rayonnements émis par l'irradiation. Au cours du fonctionnement du réacteur, les structures sont soumises à la combinaison des rayonnements neutroniques, de températures élevées et d'une exposition à un environnement corrosif. Pour une durée de fonctionnement de 60 ans, ces structures subiront des doses de plusieurs dizaines de dpa. Les structures d'internes sont des composants « remplaçables », cependant compte tenu de leur rôle dans la durabilité et de la difficulté de remplacement de l'ensemble des internes l'évaluation du comportement sous irradiation des Internes de cuve représente un enjeu économique majeur pour le Parc. Afin d'identifier, d'évaluer et d'anticiper les mécanismes de vieillissement sous irradiation des matériaux constituant ces structures, plusieurs programmes de R\&D nationaux ont été lancés depuis les années 90, en collaboration entre EDF, le CEA et AREVA. Au niveau International, plusieurs programmes ont été également lancés permettant de fédérer les données expérimentales sur les matériaux irradiés aux neutrons. Ces programmes s'appuient sur l'expertise de composants déposés et sur des irradiations menées dans des réacteurs expérimentaux. Ainsi, les phénomènes avérés de vieillissement que sont le durcissement, la perte de ductilité, le fluage d'irradiation, la fissuration sous contrainte induite par l'irradiation sont largement étudiés. Plus récemment et dans l'optique d'une durée de fonctionnement à 60 ans, des études liées à l'évaluation des conditions d'apparition du gonflement en conditions REP ont été lancées. Parallèlement à ces études expérimentales, le développement d'une modélisation prédictive des effets de l'irradiation sur l'évolution des propriétés de ces matériaux a été amorcé et a permis de fédérer les efforts de R\&D à l'échelle européenne. Les avancées liées à cette modélisation doivent rapidement permettre une meilleure compréhension des problématiques multi-mécanismes (gonflement) et multi-physiques (IASCC).

\section{6- Références}

[1] PERFORM 60 - Prediction of the effects of radiation for reactor pressure vessel and incore materials using multi-scale modelling - 60 years foreseen plant lifetime, S. Leclercq, D. Lidbury, S. Van Dyck, D. Moinereau, A. Alamo, A. Al Mazouzi, J. of Nuc. Mat., 406, pp 193-203, (2010).

[2] Irradiation creep of SA 304L and CW 316 stainless steels: Mechanical behaviour and microstructural aspects. Part II: Numerical simulation and test of SIPA model, J. Garnier, Y. Bréchet, M. Delnondedieu, A. Renault, C. Pokor, P. Dubuisson; J-P. Massoud, J. of Nuc. Mat., 413, pp 70-75, (2011)

[3] Relaxation and irradiation creep of PWR baffle bolt materials, J. Garnier, P. Dubuisson, C. Pokor, E. Lemaire, N. Monteil, J-P. Massoud, Fontevraud 7, Contribution of materials investigations to improve safety and performances of LWR's, September 26-30, 2010.

[4] Lessons learned from baffle bolt replacements regarding irradiation-induced creep behavior, E. Lemaire, N. Monteil, J.P. Massoud, C. Pokor, N. Ligneau, G. Courtemanche, Fontevraud 7, Contribution of materials investigations to improve safety and performances of LWR's, September 26-30, 2010.

[5] Corrosion sous contrainte assistée par l'irradiation des aciers inoxydables austénitiques (IASCC), B. Tanguy, Revue de Métallurgie, 108, 39-46, (2011) 
[6] Metallurgical examinations update of baffle bolts removed from operating French PWR. Microstructural investigations of a baffle to former bolt located on a high level of the internal structures. C. Panait, E. Fargeas, M. Tommy-Martin, S. Miloudi, P. Moulart, N. Monteil, C. Pokor, Fontevraud 8 - Contribution of Materials Investigations and Operating Experience to LWRs' Safety, Performance and Reliability, September 15-18, 2014.

[7] IASCC of Core Internals of PWRs : EDF R\&D and Engineering program to assess internals lifetime management, C. Pokor, C. Courtemanche, J-L. Flejou, M. Tommy-Martin, I. Rupp, B. Tanguy, J.P. Massoud N. Monteil, Fontevraud 7, Contribution of materials investigations to improve safety and performances of LWR's, September 26-30, 2010.

[8] Initiation stress threshold irradiation assisted stress corrosion cracking criterion assessment for core internals in PWR environment, B. Tanguy, C. Pokor, A. Stern, P. Bossis, Proceedings of the ASME 2011 Pressure Vessels and Piping Division Conference, PVP2011, July 17-21, 2011, Baltimore, Maryland, USA.

[9] Effect of irradiation defects on the work hardening behavior, C. Pokor, X. Averty, Y. Bréchet, P. Dubuisson; J-P. Massoud, Scripta Materiala, 50, 597-600, (2004).

[10] Irradiation damage in 304 and 316 stainless steels: experimental investigation and modeling. Part II: Irradiation induced hardening. C. Pokor, Y. Bréchet, P. Dubuisson; J-P. Massoud, , X. Averty, J. Nuc. Mater., 326, pp 30-37, (2004).

[11] Influence of irradiation temperature and dose gradients on the microstructural evolution in neutron-irradiated 316SS, D.J. Ewards, E.P. Simonen, F.A. Garner, L.R. Greenwood, B.M. Oliver, S.M. Bruemmer, J. Nuc. Mater., 317, pp 32-45, (2003).

[12] Localized deformation and IASCC initiation in austenitic stainless steels. Jiao, Z., Was, G.S., J. Nuc. Mater, 382, p. 203-209, (2008). 